\title{
Morphological and Physiological Screening of Aerial Yam (Dioscorea bulbifera L.) for Sodium Chloride Tolerance
}

\author{
S. E. Kalu ${ }^{1,2}$, A. N. Osuagwu ${ }^{1}$, I. A. Ekpo ${ }^{1}$, L. E. Okonko ${ }^{2}$ and G. M. Ubi ${ }^{1^{*}}$ \\ ${ }^{1}$ Department of Genetics and Biotechnology, University of Calabar, Calabar, Nigeria. \\ ${ }^{2}$ Department of Biological Sciences, Clifford University, Owerrinta, Nigeria.
}

\begin{abstract}
Authors' contributions
This work was carried out in collaboration among all authors. Authors SEK and ANO designed the study. Authors SEK, GMU and ANO performed the statistical analysis. Authors SEK and GMU wrote the protocol and author SKE wrote the first draft of the manuscript. Authors SEK, LEO, IAE and GMU managed the analyses of the study. Author SKE managed the literature searches. All authors read and approved the final manuscript.

Article Information

DOI: 10.9734/ARRB/2021/v36i130332 Editor(s):

(1) Dr. Rishee K. Kalaria, Navsari Agricultural University, India. Reviewers:

(1) Paula lancu, University of Craiova, Romania. (2) Ali Ammarellou, University of Zanjan, Iran. Complete Peer review History: http://www.sdiarticle4.com/review-history/61734
\end{abstract}

Original Research Article

Received 02 August 2020

Accepted 08 October 2020

Published 09 March 2021

\begin{abstract}
Aerial yam (Dioscorea bulbifera L.) is an indigenous, edible and energy-giving crop used for herbal remedy for some common ailments like diabetes, dysentery, cholera and conjunctivitis. It is fastgrowing and can adapt to different soil types and habitat conditions, therefore seen as having the potential of tolerating salt stress conditions in this area of climate change. This study, therefore, sought to determine the tolerance of $D$. bulbifera to salt stress conditions through morphological and physiological studies. Bulbils of D.bulbiferaof two distinguished shapes were sourced from local farmers in Abia State, Akwalbom State and Cross River State of the Niger- Delta region of Nigeria, and established in Biological Sciences Research Farm, University of Calabar, Calabar, to have the various lines for the experiment. Randomized Complete Block Design (RCBD) with three replications was used for the study. At four weeks after sprouting, the plants were treated with $250 \mathrm{ml}$ of different concentrations of sodium chloride $(0,100,200$ and $300 \mathrm{mM})$ twice a week for six weeks. Morphological data of leaf color, number of leaves/plant, root length, leaf length, petiole
\end{abstract}


length, leaf area, vine length and number of bulbils were measured weekly during the treatment period while physiological data of Chlorophyll-a and chlorophyll- b concentrations, peroxidase and glucose-6-phosphate dehydrogenase activities were analyzed two weeks after the completion of the treatment. Results revealed that sodium chloride reduced the vine length, number of leaves, number of bulbils, chlorophyll-a and chlorophyll-b concentrations of the plant, and caused an increase in the root length, peroxidase and glucose-6- phosphate dehydrogenase activities. Although sodium chloride reduced the growth of the D.bulbifera, all the lines were able to produce bulbils at a salt concentration of $0.00 \mathrm{mM}$ to $200 \mathrm{mM}$. Therefore, this plantcould be regarded as a moderate salt tolerant plant. Significant differences in growth and yield were found between the lines $(P<0.05)$. Bulbils with round shape tended to show more growth than the spindle shape.

Keywords: Yam (Dioscorea bulbifera L.); sodium chloride; tolerance; physiological; growth and yield.

\section{INTRODUCTION}

Environmental deterioration caused by human activities such as deforestation, irrigation practices, bush burning and excessive use of agrochemicals has added so much salt to the topsoil and the root zone of plants and has thus resulted in abiotic stresses in plants [1].

Abiotic stress contributes to the low productivity of crops [2]. It has been estimated that about $70 \%$ of the step down in yields of crop plants is as a result of abiotic stress. Salinity, a major abiotic stressor has affected an estimated 45 million hectares of irrigated land and is expected to increase due to climate change [3]. [4] has estimated that about $50 \%$ of presently cultivated lands would be lost by the year 2050 if salt stress persists.

Soil salinity is an environmental problem affecting agriculture [5] and has greatly contributed to the low productivity of plants worldwide $[6,7]$. Saline soil causes physiological and metabolic imbalance resulting in poor development, growth and yield of plants [8].

There is a consensus among researchers that salinity harms the morphology of plants and ultimately on its yield and productivity of which include dehydration and generation of reactive oxygen species like hydrogen peroxide which may cause cell damage and retarded growth. [9] reported a decrease in the total root length of $E$. japonica treated with salt. [10] also reported the same decrease in Echiumamoenum. Sodium chloride was observed to cause a loss in the fresh root weight of maize, an effect that increases with a higher level of salinity [11]. [12] reported a decrease in leaf area and root length with salinity in Salvinianatans. Salinity also causes a reduction in chlorophyll concentrations of plants [13].
Despite the reduction in growth and yield of crop plants by salinity, some species of plants such as rice and maize are reported to have the capacity of tolerating salinity in various concentrations. This ability to tolerate salinity is made possible through an increase in the activities of enzymes associated with salt stress $[14,15]$. The increased enzyme activity acts as a defensive mechanism against cell damage and contributes to the tolerance response of plants [15].

Aerial yam (Dioscorea bulbifera) is an indigenous edible crop cultivated for its cheap source of the nutrient. It is fast-growing and can adapt to different soil types, weather and habitat conditions, therefore having the potential of tolerating salt stress thus providing food security in the era of famine occasioned by climate change.

This study, therefore, aimed to evaluate the tolerance of aerial yam to sodium chloride through morphological and physiological studies.

\section{MATERIALS AND METHODS}

\subsection{Collection and Experimental Materials}

Bulbils of $D$. bulbifera with two distinguished shapes were obtained from local farmers in Abia State, Akwalbom State and Cross River State and established in the Biological Sciences Research Farm, University of Calabar, Calabar, to have the various lines for the study. Sodium chloride and other reagents were purchased from a commercial chemical store. All reagents were of analytical grade.

\subsection{Planting and Treatment}

Bulbils were planted in polyethylene bags and placed in a greenhouse in a randomized 
complete block design with three replicates. Three bulbils were planted per bag and watered with $250 \mathrm{ml}$ of distilled water daily until sprouting was established after which the plants were reduced to one per bag. At four weeks after sprouting, the plants were treated with $250 \mathrm{ml}$ of various concentrations of sodium chloride solution $(0,100,200$ and $300 \mathrm{mM})$ twice a week for six weeks during which morphological data of vine length, number of leaves per plant, leaf area, petiole length and leaf length were measured weekly. Physiological indices such as chlorophyll-a and b concentrations, peroxidase and glucose-6 phosphate dehydrogenase activities were measured spectrophotometrically from leaf extracts of the plant samples.

\subsection{Extracts Preparation}

Leaf samples were weighed, frozen and ground using mortar and pestle. Grounded samples were hydrated with $20 \mathrm{ml}$ of $80 \%$ acetone and phosphate buffer for chlorophyll and enzyme's activities respectively. Each of the mixtures was incubated and centrifuged at 15000 rpm for four minutes. Supernatants were collected and stored on ice until further studies.

\subsection{Chlorophyll Concentration Assay}

Each of the sample extracts was transferred into a cuvette and absorbance read at a wavelength of $643 \mathrm{~nm}$ and $663 \mathrm{~nm}$. Chlorophyll concentrations were calculated from the formula as described by Strickland and Parsons (1972).

\subsection{Peroxidase Activity Assay}

The extract $(0.6 \mathrm{ml})$, hydrogen peroxide $(0.1 \mathrm{ml})$, guaiacol $(0.1 \mathrm{ml})$ and buffer $(2 \mathrm{ml})$ were added together in a cuvette for a reaction and the absorbance value was read at a wavelength of $436 \mathrm{~nm}$ after one minute, two, three, four and five minutes. The values were used to determine the peroxidase activity.

\subsection{Glucose- 6 Phosphate Dehydrogenase Activity Assay}

The reaction mixture for glucose- 6 phosphate dehydrogenase activity included $0.6 \mathrm{ml}$ extract, $2.0 \mathrm{ml}$ buffer, $0.1 \mathrm{ml}$ glucose -6 phosphate and $0.1 \mathrm{ml}$ beta Nicotinamide adenine dinucleotide phosphate. The absorbance values were read at a wavelength of $340 \mathrm{~nm}$ at one minute, two, three, four and five minutes. The values were used to determine the glucose- 6 phosphate dehydrogenase activity.

\subsection{Data Analysis}

Analysis of variance (ANOVA) was used to analyze the data and significant differences were calculated using the least significant difference (LSD).

\section{RESULTS AND DISCUSSION}

The results of sodium chloride effect on the chlorophyll a and b, enzymes activities and morphology parameters in Dioscorea bulbifera are shown in Tables 1 - 3.

Sodium chloride was observed that increase the glucose-6 phosphate dehydrogenase and peroxidase activities in $D$. bulbifera. An increase in these enzyme activities suggests the formation of excess hydrogen peroxide by the salt and eventually oxidative stress. Therefore, as a defense response to the oxidative stress, the peroxidase and glucose- 6 phosphate dehydrogenase activities were increased. This agrees with $[13,15]$, who reported an increase in anti-oxidative enzymes under salt stress.

Sodium chloride reduced the morphological parameters in Dioscorea bulbifera as enlisted in Table 2. Specifically, it decreases the vine length, number of leaves and number of bulbils of the $D$. bulbifera.At $100 \mathrm{mM}$, the height and number of leaves of $D$. bulbifera of some of the lines were at a higher value, although not significantly different from that of the controls. From this, it can be deduced that a higher concentration of sodium chloride (>100mM) caused a reduction in the growth of $D$. bulbiferaand the lower concentration of sodium chloride $(\leq 100 \mathrm{mM})$ tend to stimulate the growth of $D$. bulbifera. The reduction in the growth of Dioscorea bulbifera by sodium chloride agrees with authors who reported a reduction in growth and yield of plants exposed to salt stress. [14,15] reported a reduction in the growth of Euryaemarginata by salt stress as evidenced by reduction in fresh weight, leaf water content and chlorophyll a and b content.

The root length of the treated plants showed a significant increase at $200 \mathrm{mM}$ and $300 \mathrm{mM}$ when compared to the control. This buttressed the fact that a higher concentration of sodium chloride (>100 mM) may interfere with the absorption of 
Table 1. Chlorophyll -a and b concentrations in leaves extracts of Dioscorea bulbifera treated with different concentrations of sodium chloride

\begin{tabular}{|c|c|c|c|c|c|c|c|c|c|c|c|c|}
\hline Parameter & $0.00 \mathrm{mM}$ & $\begin{array}{l}\text { Line } 1 \\
100 \mathrm{mM}\end{array}$ & $200 \mathrm{mM}$ & $300 \mathrm{mM}$ & $0.00 \mathrm{mM}$ & $\begin{array}{l}\text { Line } 2 \\
100 \mathrm{mM}\end{array}$ & $200 \mathrm{mM}$ & $300 \mathrm{mM}$ & $0.00 \mathrm{mM}$ & $\begin{array}{l}\text { Line } 3 \\
100 \mathrm{mM}\end{array}$ & $200 \mathrm{mM}$ & $300 \mathrm{mM}$ \\
\hline Chlo.-a $\left(\mathrm{mgg}^{-1}\right) \mathrm{FW}$ & $139.70^{\mathrm{a}}$ & $139.90^{a}$ & $136.20^{b}$ & $134.60^{\mathrm{C}}$ & $144.30^{a}$ & $144.00^{a}$ & $139.60^{b}$ & $137.40^{C}$ & $143.40^{a}$ & $143.70^{a}$ & $138.20^{b}$ & $137.80^{c}$ \\
\hline Chlo.-b $\left(\mathrm{mgg}^{-1}\right) \mathrm{FW}$ & $192.10^{\mathrm{a}}$ & $191.30^{a}$ & $140.80^{\mathrm{b}}$ & $34.70^{c}$ & $189.80^{\mathrm{a}}$ & $189.20^{\mathrm{a}}$ & $140.70^{b}$ & $34.10^{\mathrm{C}}$ & $196.00^{\mathrm{a}}$ & $195.40^{a}$ & $143.60^{b}$ & $32.90^{\mathrm{C}}$ \\
\hline & & \multicolumn{2}{|l|}{ Line 4} & & & \multicolumn{2}{|l|}{ Line 5} & & & \multicolumn{2}{|l|}{ Line 6} & \\
\hline Chlo.-a $\left(\mathrm{mgg}^{-1}\right) \mathrm{FW}$ & $144.20^{a}$ & $144.00^{a}$ & $139.40^{b}$ & $138.00^{c}$ & $146.20^{a}$ & $146.00^{\mathrm{a}}$ & $142.60^{b}$ & $139.70^{C}$ & $146.80^{\mathrm{a}}$ & \multirow{2}{*}{$\begin{array}{l}146.4^{\mathrm{a}} \\
206.70^{\mathrm{a}}\end{array}$} & $143.3^{b}$ & $139.2^{c}$ \\
\hline \multirow[t]{2}{*}{ Chlo.-b $\left(\mathrm{mgg}^{-1}\right) \mathrm{FW}$} & $187.70^{\mathrm{a}}$ & $187.10^{\mathrm{a}}$ & $140.20^{b}$ & $31.20^{c}$ & $204.40^{\mathrm{a}}$ & $203.60^{a}$ & $166.70^{\mathrm{b}}$ & $73.40^{\mathrm{c}}$ & $206.90^{\mathrm{a}}$ & & $168.20^{b}$ & $75.20^{\mathrm{C}}$ \\
\hline & & \multicolumn{3}{|l|}{ Line 7} & & \multicolumn{2}{|l|}{ Line 8} & & & \multicolumn{2}{|l|}{ Line 9} & \\
\hline Chlo.-a $\left(\mathrm{mgg}^{-1}\right) \mathrm{FW}$ & $146.50^{a}$ & $146.20^{a}$ & $142.70^{b}$ & $139.90^{C}$ & $147.10^{\mathrm{a}}$ & $146.40^{b}$ & $144.30^{c}$ & $140.80^{d}$ & $152.10^{\mathrm{a}}$ & \multirow{2}{*}{$\begin{array}{l}152.30^{\mathrm{a}} \\
212.90^{\mathrm{a}}\end{array}$} & $147.90^{b}$ & $143.90^{c}$ \\
\hline Chlo.-b $\left(\mathrm{mgg}^{-1}\right) \mathrm{FW}$ & $207.60^{\mathrm{a}}$ & $207.60^{a}$ & $166.90^{\mathrm{b}}$ & $74.60^{\mathrm{C}}$ & $206.20^{\mathrm{a}}$ & $206.40^{\mathrm{a}}$ & $168.40^{\mathrm{b}}$ & $74.90^{\mathrm{C}}$ & $213.70^{\mathrm{a}}$ & & $179.60^{\mathrm{b}}$ & $79.80^{\mathrm{C}}$ \\
\hline Parameter & $0.00 \mathrm{mM}$ & $\begin{array}{l}\text { Line } 10 \\
100 \mathrm{mM}\end{array}$ & $200 \mathrm{mM}$ & $300 \mathrm{mM}$ & $0.00 \mathrm{mM}$ & $\begin{array}{l}\text { Line } 11 \\
100 \mathrm{mM}\end{array}$ & $200 \mathrm{mM}$ & $300 \mathrm{mM}$ & $0.00 \mathrm{mM}$ & $\begin{array}{l}\text { Line } 12 \\
100 \mathrm{mM}\end{array}$ & $200 \mathrm{mM}$ & $300 \mathrm{mM}$ \\
\hline Chlo.-a $\left(\mathrm{mgg}^{-1}\right) \mathrm{FW}$ & $151.90^{\mathrm{a}}$ & $151.50^{a}$ & $147.90^{b}$ & $143.40^{\mathrm{C}}$ & $152.40^{a}$ & $152.50^{\mathrm{a}}$ & $148.50^{b}$ & $144.30^{c}$ & $152.70^{a}$ & $152.80^{\mathrm{a}}$ & $148.30^{b}$ & $144.20^{C}$ \\
\hline Chlo.-b $\left(\mathrm{mgg}^{-1}\right) \mathrm{FW}$ & $212.90^{\mathrm{b}}$ & $219.90^{a}$ & $182.30^{\mathrm{C}}$ & $82.20^{d}$ & $217.30^{\mathrm{a}}$ & $216.10^{a}$ & $179.90^{b}$ & $87.10^{c}$ & $214.20^{\mathrm{a}}$ & $214.00^{a}$ & $176.80^{b}$ & $85.70^{c}$ \\
\hline & & \multicolumn{3}{|l|}{ Line 13} & & Line 14 & & & & \multicolumn{2}{|l|}{ Line 15} & \\
\hline Chlo.-a $\left(\mathrm{mgg}^{-1}\right) \mathrm{FW}$ & $155.80^{\mathrm{a}}$ & $155.70^{a}$ & $152.90^{b}$ & $148.00^{C}$ & $157.10^{\mathrm{a}}$ & $157.30^{a}$ & $154.10^{b}$ & $149.40^{c}$ & $154.90^{\mathrm{a}}$ & $154.40^{b}$ & $152.80^{C}$ & $147.80^{d}$ \\
\hline \multirow[t]{2}{*}{ Chlo.-b $\left(\mathrm{mgg}^{-1}\right) \mathrm{FW}$} & $219.20^{a}$ & $218.80^{a}$ & $211.00^{b}$ & $99.80^{\mathrm{C}}$ & $214.80^{\mathrm{a}}$ & $213.90^{a}$ & $209.80^{b}$ & $99.20^{\mathrm{C}}$ & $212.40^{\mathrm{a}}$ & $212.40^{a}$ & $210.10^{b}$ & $98.60^{c}$ \\
\hline & & \multicolumn{3}{|l|}{ Line 16} & & Line 17 & & & & \multicolumn{2}{|l|}{ Line 18} & \\
\hline Chlo.-a $\left(\mathrm{mgg}^{-1}\right) \mathrm{FW}$ & $155.20^{\mathrm{a}}$ & $155.60^{a}$ & $153.40^{b}$ & $149.00^{c}$ & $204.10^{a}$ & $204.40^{\mathrm{a}}$ & $199.60^{b}$ & $192.40^{c}$ & $204.20^{\mathrm{a}}$ & \multirow{2}{*}{$\begin{array}{l}204.00^{a} \\
231.90^{a}\end{array}$} & $199.00^{b}$ & $193.70^{\mathrm{C}}$ \\
\hline Chlo.-b $\left(\mathrm{mgg}^{-1}\right) \mathrm{FW}$ & $216.20^{\mathrm{a}}$ & $215.80^{a}$ & $210.70^{b}$ & $99.20^{\mathrm{C}}$ & $230.90^{\mathrm{a}}$ & $230.20^{\mathrm{a}}$ & $223.30^{\mathrm{b}}$ & $102.20^{c}$ & $232.40^{\mathrm{a}}$ & & $223.80^{b}$ & $103.70^{c}$ \\
\hline Parameter & $0.00 \mathrm{mM}$ & \multicolumn{2}{|c|}{$\begin{array}{l}\text { Line } 19 \\
100 \mathrm{mM}\end{array}$} & $200 \mathrm{mM}$ & \multicolumn{2}{|c|}{$300 \mathrm{mM}$} & 00mM & $\begin{array}{l}\text { Line } 20 \\
100 \mathrm{mM}\end{array}$ & \multicolumn{2}{|c|}{$200 \mathrm{mM}$} & $300 \mathrm{mM}$ & LSD \\
\hline Chlo.-a $\left(\mathrm{mgg}^{-1}\right) \mathrm{FW}$ & $204.40 a$ & \multirow{2}{*}{\multicolumn{2}{|c|}{$\begin{array}{l}204.10 a \\
23270^{a}\end{array}$}} & $201.1 b$ & \multirow{2}{*}{\multicolumn{2}{|c|}{$\begin{array}{l}194.50 c \\
102.60 c\end{array}$}} & $04.20 a$ & $204.10 a$ & \multirow{2}{*}{\multicolumn{2}{|c|}{$\begin{array}{l}199.40 b \\
224.40 b\end{array}$}} & \multirow{2}{*}{$\begin{array}{l}94.00 \mathrm{c} \\
04.90 \mathrm{c}\end{array}$} & 0.51 \\
\hline Chlo.-b $\left(\mathrm{mgg}^{-1}\right) \mathrm{FW}$ & $232.80^{\mathrm{a}}$ & & & $223.80 b$ & & & $32.50 a$ & $232.20 \mathrm{a}$ & & & & 5.40 \\
\hline
\end{tabular}

Means with the same superscripts on the same row are not significantly different $(p<0.05)$ 
Table 2. Enzyme's activities in leaves extract of Dioscorea bulbifera treated with different concentrations of sodium chloride

\begin{tabular}{|c|c|c|c|c|c|c|c|c|c|c|c|c|}
\hline Enzyme & $0.00 \mathrm{mM}$ & $\begin{array}{l}\text { Line1 } \\
\text { 100mM }\end{array}$ & $200 \mathrm{mM}$ & $300 \mathrm{mM}$ & $0.00 \mathrm{mM}$ & $\begin{array}{l}\text { Line2 } \\
\text { 100mM }\end{array}$ & $200 \mathrm{mM}$ & $300 \mathrm{mM}$ & $0.00 \mathrm{mM}$ & $\begin{array}{l}\text { Line } 3 \\
100 \mathrm{mM}\end{array}$ & $200 \mathrm{mM}$ & $300 \mathrm{mM}$ \\
\hline G6PD & $0.01^{\mathrm{d}}$ & $0.02^{c}$ & $0.04^{b}$ & $0.06^{a}$ & $0.01 d$ & $0.03 c$ & $0.05 b$ & $0.06 a$ & $0.01^{\mathrm{d}}$ & $0.02^{\mathrm{C}}$ & $0.04^{b}$ & $0.06^{a}$ \\
\hline \multirow[t]{2}{*}{ Peroxidase } & $0.01^{d}$ & $0.02^{\mathrm{C}}$ & $0.03^{b}$ & $0.04^{a}$ & $0.01^{c}$ & $0.02^{\mathrm{b}}$ & $0.03^{a}$ & $0.03^{a}$ & $0.01^{d}$ & $0.02^{\mathrm{C}}$ & $0.03^{b}$ & $0.04^{a}$ \\
\hline & & Line 4 & & & & Line 5 & & & & LSD $\times 10^{-1}$ & & \\
\hline G6PD & $1.51^{\mathrm{d}}$ & $1.53^{\mathrm{C}}$ & $1.54^{b}$ & $1.56^{\mathrm{a}}$ & $1.50 d$ & $1.52 \mathrm{c}$ & $1.54 \mathrm{~b}$ & $1.55 a$ & & 0.03 & & \\
\hline Peroxidase & $0.01^{\mathrm{c}}$ & $0.02^{\mathrm{b}}$ & $0.03^{a}$ & $0.03^{a}$ & $0.01^{\mathrm{c}}$ & $0.01^{\mathrm{c}}$ & $0.02^{\mathrm{b}}$ & $0.03^{a}$ & & & & \\
\hline
\end{tabular}

Table 3a. Morphological parameters of Dioscorea bulbifera treated with different concentrations of sodium chloride

\begin{tabular}{|c|c|c|c|c|c|c|c|c|c|c|c|c|}
\hline Parameter & $\begin{array}{l}\text { Line } 1 \\
0.00 \mathrm{mM}\end{array}$ & $100 \mathrm{mM}$ & $200 \mathrm{mM}$ & $300 \mathrm{Mm}$ & $\begin{array}{l}\text { Line } 2 \\
0.00 \mathrm{mM}\end{array}$ & $100 \mathrm{mM}$ & $200 \mathrm{mM}$ & $300 \mathrm{mM}$ & $\begin{array}{l}\text { Line } 3 \\
0.00 \mathrm{mM}\end{array}$ & $100 \mathrm{mM}$ & $200 \mathrm{mM}$ & $300 \mathrm{mM}$ \\
\hline No of leaves & $25.00^{\mathrm{a}}$ & $24.00^{b}$ & $22.00^{\mathrm{C}}$ & $19.00^{d}$ & $25.00^{a}$ & $24.00^{b}$ & $21.00^{C}$ & $19.00^{d}$ & $25.00^{b}$ & $27.00^{\mathrm{a}}$ & $21.00^{C}$ & $18.00^{\mathrm{d}}$ \\
\hline Root length $(\mathrm{cm})$ & $13.00^{c}$ & $13.20^{\mathrm{c}}$ & $15.00^{b}$ & $17.00^{a}$ & $13.40^{\mathrm{C}}$ & $13.50^{\mathrm{C}}$ & $14.50^{\mathrm{b}}$ & $15.90^{a}$ & $12.80^{\mathrm{C}}$ & $13.00^{c}$ & $13.90^{\mathrm{b}}$ & $15.60^{\mathrm{a}}$ \\
\hline Leaf length (cm) & $8.35^{\mathrm{a}}$ & $8.35^{\mathrm{a}}$ & $8.40^{\mathrm{a}}$ & $8.40^{\mathrm{a}}$ & $8.00^{\mathrm{a}}$ & $8.00^{\mathrm{a}}$ & $8.00^{a}$ & $8.00^{\mathrm{a}}$ & $8.15^{\mathrm{a}}$ & $8.15^{\mathrm{a}}$ & $8.13^{\mathrm{a}}$ & $8.13^{a}$ \\
\hline Petiole length $(\mathrm{cm})$ & $5.73^{a}$ & $5.65^{a}$ & $5.63^{a}$ & $5.60^{a}$ & $5.65^{\mathrm{a}}$ & $5.63^{a}$ & $5.53^{\mathrm{a}}$ & $5.63^{\mathrm{a}}$ & $5.48^{\mathrm{a}}$ & $5.58^{\mathrm{a}}$ & $5.58^{\mathrm{a}}$ & $5.53^{\mathrm{a}}$ \\
\hline Leaf area $\left(\mathrm{cm}^{2}\right)$ & $67.50^{a}$ & $66.75^{\mathrm{a}}$ & $66.75^{\mathrm{a}}$ & $67.00^{\mathrm{a}}$ & $66.75^{\mathrm{a}}$ & $67.00^{a}$ & $66.75^{\mathrm{a}}$ & $66.50^{\mathrm{a}}$ & $67.25^{\mathrm{a}}$ & $67.50^{a}$ & $67.25^{\mathrm{a}}$ & $67.25^{\mathrm{a}}$ \\
\hline Vine length $(\mathrm{cm})$ & $180.25^{\mathrm{a}}$ & $176.00^{\mathrm{b}}$ & $163.25^{c}$ & $151.00^{\mathrm{d}}$ & $183.00^{\mathrm{a}}$ & $174.00^{\mathrm{b}}$ & $167.00^{c}$ & $157.00^{\mathrm{d}}$ & $188.25^{\mathrm{b}}$ & $192.00^{\mathrm{a}}$ & $169.25^{\mathrm{c}}$ & $157.25^{\mathrm{d}}$ \\
\hline No of bulbils & $2.00^{\mathrm{a}}$ & $2.00^{\mathrm{a}}$ & $2.00^{\mathrm{a}}$ & $0.00^{\mathrm{b}}$ & $2.00^{\mathrm{b}}$ & $3.00^{\mathrm{a}}$ & $2.00^{\mathrm{b}}$ & $0.00^{c}$ & $3.00^{\mathrm{a}}$ & $3.00^{\mathrm{a}}$ & $2.00^{\mathrm{b}}$ & $0.00^{c}$ \\
\hline Parameter & $\begin{array}{l}\text { Line } 4 \\
0.00 \mathrm{mM}\end{array}$ & $100 \mathrm{mM}$ & $200 \mathrm{mM}$ & $300 \mathrm{mM}$ & $\begin{array}{l}\text { Line } 5 \\
0.00 \mathrm{mM}\end{array}$ & $100 \mathrm{mM}$ & $200 \mathrm{mM}$ & $300 \mathrm{mM}$ & $\begin{array}{l}\text { Line } 6 \\
0.00 \mathrm{mM}\end{array}$ & $100 \mathrm{mM}$ & $200 \mathrm{mM}$ & $300 \mathrm{mM}$ \\
\hline No of leaves & $24.00^{a}$ & $24.00^{\mathrm{a}}$ & $20.00^{b}$ & $17.00^{\mathrm{C}}$ & $25.00^{\mathrm{a}}$ & $23.00^{b}$ & $20.00^{C}$ & $18.00^{\mathrm{d}}$ & $25.00^{\mathrm{a}}$ & $24.00^{\mathrm{b}}$ & $20.00^{C}$ & $17.00^{\mathrm{d}}$ \\
\hline Root length $(\mathrm{cm})$ & $13.00^{\mathrm{C}}$ & $13.00^{\mathrm{c}}$ & $14.60^{\mathrm{b}}$ & $15.40^{\mathrm{a}}$ & $13.60^{\mathrm{C}}$ & $13.70^{\mathrm{C}}$ & $14.50^{\mathrm{b}}$ & $15.30^{\mathrm{b}}$ & $13.90^{\mathrm{C}}$ & $14.00^{\mathrm{c}}$ & $14.80^{\mathrm{b}}$ & $16.30^{a}$ \\
\hline Leaf length $(\mathrm{cm})$ & $7.95^{\mathrm{a}}$ & $7.93^{\mathrm{a}}$ & $7.93^{\mathrm{a}}$ & $7.93^{\mathrm{a}}$ & $8.08^{a}$ & $8.03^{\mathrm{d}}$ & $8.03^{\mathrm{a}}$ & $8.00^{a}$ & $8.18^{a}$ & $8.15^{\mathrm{a}}$ & $8.15^{\mathrm{a}}$ & $8.13^{\mathrm{a}}$ \\
\hline Petiole length $(\mathrm{cm})$ & $5.68^{a}$ & $5.55_{a}$ & $5.58^{\mathrm{a}}$ & $5.55^{\mathrm{a}}$ & $4.55^{\mathrm{a}}$ & $4.60^{a}$ & $4.55^{\mathrm{a}}$ & $4.60^{\mathrm{a}}$ & $4.50^{\mathrm{a}}$ & $4.58^{\mathrm{a}}$ & $4.50^{\mathrm{a}}$ & $4.60^{\mathrm{a}}$ \\
\hline Leaf area $\left(\mathrm{cm}^{2}\right)$ & $67.00^{\mathrm{a}}$ & $66.75^{a}$ & $66.75^{\mathrm{a}}$ & $66.75^{a}$ & $61.00^{a}$ & $60.75^{\mathrm{a}}$ & $60.75^{\mathrm{a}}$ & $60.75^{a}$ & $61.25^{\mathrm{a}}$ & $61.25^{\mathrm{a}}$ & $61.25^{\mathrm{a}}$ & $60.75^{\mathrm{a}}$ \\
\hline Vine length $(\mathrm{cm})$ & $184.50^{a}$ & $182.75^{a}$ & $167.75^{b}$ & $159.00^{c}$ & $185.75^{\mathrm{a}}$ & $182.25^{\mathrm{b}}$ & $169.00^{c}$ & $157.75^{d}$ & $192.50^{a}$ & $186.75^{\mathrm{b}}$ & $173.00^{c}$ & $162.00^{d}$ \\
\hline No of bulbils & $3.00^{\mathrm{a}}$ & $3.00^{\mathrm{a}}$ & $1.00^{\mathrm{b}}$ & $0.00^{c}$ & $4.00^{\mathrm{a}}$ & $4.00^{\mathrm{a}}$ & $4.00^{\mathrm{a}}$ & $1.00^{\mathrm{b}}$ & $3.00^{\mathrm{a}}$ & $3.00^{\mathrm{a}}$ & $2.00^{\mathrm{b}}$ & $0.00^{c}$ \\
\hline
\end{tabular}


Table 3b. Morphological parameters of Dioscorea bulbifera treated with different concentrations of sodium chloride

\begin{tabular}{|c|c|c|c|c|c|c|c|c|c|c|c|c|}
\hline Parameter & $\begin{array}{l}\text { Line } 7 \\
0.00 \mathrm{mM}\end{array}$ & $100 \mathrm{mM}$ & $200 \mathrm{mM}$ & $300 \mathrm{mM}$ & $\begin{array}{l}\text { Line } 8 \\
0.00 \mathrm{mM}\end{array}$ & $100 \mathrm{mM}$ & $200 \mathrm{mM}$ & $300 \mathrm{mM}$ & $\begin{array}{l}\text { Line } 9 \\
0.00 \mathrm{mM}\end{array}$ & $100 \mathrm{mM}$ & $200 \mathrm{mM}$ & $300 \mathrm{mM}$ \\
\hline No of leaves & $32.00^{a}$ & $32.00^{\mathrm{a}}$ & $28.00^{b}$ & $24.00^{C}$ & $31.00^{\mathrm{a}}$ & $30.00^{b}$ & $28.00^{c}$ & $23.00^{d}$ & $30.00^{b}$ & $31.00^{a}$ & $27.00^{\mathrm{C}}$ & $24.00^{d}$ \\
\hline Root length (cm) & $14.10^{c}$ & $14.10^{c}$ & $14.90^{\mathrm{b}}$ & $16.40^{\mathrm{a}}$ & $14.20^{\mathrm{C}}$ & $14.50^{c}$ & $15.60^{b}$ & $16.50^{a}$ & $17.00 \mathrm{~d}$ & $14.50 \mathrm{c}$ & $15.60 \mathrm{~b}$ & $16.50 d$ \\
\hline Leaf length (cm) & $8.23^{a}$ & $8.18^{a}$ & $8.18^{\mathrm{a}}$ & $8.15^{\mathrm{a}}$ & $8.28^{a}$ & $8.23^{a}$ & $8.25^{\mathrm{a}}$ & $8.23^{\mathrm{a}}$ & $8.75^{\mathrm{a}}$ & $8.25^{\mathrm{a}}$ & $8.25^{\mathrm{a}}$ & $8.25^{\mathrm{a}}$ \\
\hline Petiole length $(\mathrm{cm})$ & $4.50^{\mathrm{a}}$ & $4.70^{a}$ & $4.48^{\mathrm{a}}$ & $4.55^{\mathrm{a}}$ & $4.55^{\mathrm{a}}$ & $4.55^{\mathrm{a}}$ & $4.55^{\mathrm{a}}$ & $4.60^{a}$ & $8.60^{a}$ & $8.60^{a}$ & $8.60^{a}$ & $8.60^{a}$ \\
\hline Leaf area $\left(\mathrm{cm}^{2}\right)$ & $60.75^{\mathrm{a}}$ & $61.00^{a}$ & $60.75^{\mathrm{a}}$ & $60.75^{\mathrm{a}}$ & $61.00^{a}$ & $61.00^{a}$ & $60.75^{\mathrm{a}}$ & $60.75^{a}$ & $70.50^{a}$ & $70.75^{\mathrm{a}}$ & $70.50^{a}$ & $70.50^{a}$ \\
\hline Vine length $(\mathrm{cm})$ & $191.75^{a}$ & $190.00^{a}$ & $169.75^{b}$ & $159.75^{c}$ & $194.25^{\mathrm{a}}$ & $192.00^{\mathrm{b}}$ & $176.25^{\mathrm{C}}$ & $165.00^{d}$ & $254.00^{a}$ & $256.00^{a}$ & $232.00^{\mathrm{b}}$ & $220.25^{c}$ \\
\hline No of bulbils & $4.00^{a}$ & $3.00^{\mathrm{b}}$ & $3.00^{\mathrm{b}}$ & $2.00^{c}$ & $3.00^{\mathrm{b}}$ & $4.00^{a}$ & $2.00^{c}$ & $1.00^{d}$ & $4.00^{a}$ & $4.00^{a}$ & $3.00^{\mathrm{b}}$ & $0.00^{c}$ \\
\hline Parameter & $\begin{array}{l}\text { Line } 10 \\
0.00 \mathrm{mM}\end{array}$ & $100 \mathrm{mM}$ & $200 \mathrm{mM}$ & $300 \mathrm{mM}$ & $\begin{array}{l}\text { Line } 11 \\
0.00 \mathrm{mM}\end{array}$ & $100 \mathrm{mM}$ & $200 \mathrm{mM}$ & $300 \mathrm{mM}$ & $\begin{array}{l}\text { Line } 12 \\
0.00 \mathrm{mM}\end{array}$ & $100 \mathrm{mM}$ & $200 \mathrm{mM}$ & $300 \mathrm{mM}$ \\
\hline No of leaves & $31.00^{a}$ & $31.00^{\mathrm{a}}$ & $28.00^{b}$ & $25.00^{c}$ & $32.00^{\mathrm{a}}$ & $32.00^{\mathrm{a}}$ & $29.00^{b}$ & $25.00^{c}$ & $31.00^{\mathrm{a}}$ & $30.00^{b}$ & $26.00^{c}$ & $24.00^{d}$ \\
\hline Root length $(\mathrm{cm})$ & $15.80^{\mathrm{C}}$ & $16.00^{c}$ & $20.00^{b}$ & $23.10^{a}$ & $16.00^{c}$ & $16.40^{c}$ & $21.00^{b}$ & $23.70^{a}$ & $16.20^{c}$ & $16.60^{c}$ & $21.00^{b}$ & $24.00^{a}$ \\
\hline Leaf length $(\mathrm{cm})$ & $8.00^{a}$ & $8.00^{\mathrm{a}}$ & $8.00^{\mathrm{a}}$ & $8.00^{\mathrm{a}}$ & $8.00^{\mathrm{a}}$ & $8.00^{\mathrm{a}}$ & $8.00^{\mathrm{a}}$ & $8.75^{a}$ & $8.95^{\mathrm{a}}$ & $8.90^{\mathrm{a}}$ & $8.90^{\mathrm{a}}$ & $8.75^{a}$ \\
\hline Petiole length $(\mathrm{cm})$ & $8.55^{\mathrm{a}}$ & $8.55^{\mathrm{a}}$ & $8.60^{\mathrm{a}}$ & $8.60^{\mathrm{a}}$ & $8.55^{\mathrm{a}}$ & $8.45^{\mathrm{a}}$ & $8.58^{a}$ & $8.53^{\mathrm{a}}$ & $8.40^{\mathrm{a}}$ & $8.60^{\mathrm{a}}$ & $8.60^{\mathrm{a}}$ & $8.60^{\mathrm{a}}$ \\
\hline Leaf area $\left(\mathrm{cm}^{2}\right)$ & $71.25^{\mathrm{a}}$ & $71.25^{\mathrm{a}}$ & $71.25^{\mathrm{a}}$ & $71.25^{\mathrm{a}}$ & $70.75^{\mathrm{a}}$ & $71.50^{\mathrm{a}}$ & $70.50^{a}$ & $70.75^{a}$ & $72.00^{a}$ & $72.00^{a}$ & $71.50^{\mathrm{a}}$ & $71.25^{\mathrm{a}}$ \\
\hline Vine length (cm) & $240.50^{a}$ & $238.75^{a}$ & $228.25^{\mathrm{b}}$ & $220.00^{c}$ & $252.25^{a}$ & $254.00^{a}$ & $234.75^{\mathrm{b}}$ & $218.00^{c}$ & $243.25^{\mathrm{a}}$ & $245.00^{a}$ & $229.00^{b}$ & $215.00^{c}$ \\
\hline No of bulbils & $3.00^{\mathrm{a}}$ & $3.00^{a}$ & $3.00^{a}$ & $0.00^{\mathrm{b}}$ & $4.00^{\mathrm{a}}$ & $3.00^{\mathrm{b}}$ & $3.00^{\mathrm{b}}$ & $0.00^{c}$ & $3.00^{\mathrm{b}}$ & $4.00^{a}$ & $2.00^{\mathrm{C}}$ & $1.00^{\mathrm{d}}$ \\
\hline Parameter & $\begin{array}{l}\text { Line } 13 \\
0.00 \mathrm{mM}\end{array}$ & $100 \mathrm{mM}$ & $200 \mathrm{mM}$ & $300 \mathrm{mM}$ & $\begin{array}{l}\text { Line } 14 \\
0.00 \mathrm{mM}\end{array}$ & $100 \mathrm{mM}$ & $200 \mathrm{mM}$ & $300 \mathrm{mM}$ & $\begin{array}{l}\text { Line } 15 \\
\text { 0.00mM }\end{array}$ & $100 \mathrm{mM}$ & $200 \mathrm{mM}$ & $300 \mathrm{mM}$ \\
\hline No of leaves & $36.00^{a}$ & $36.00^{a}$ & $30.00^{b}$ & $27.00^{c}$ & $39.00^{a}$ & $30.00^{\mathrm{a}}$ & $32.00^{b}$ & $29.00^{c}$ & $38.00^{a}$ & $37.00^{b}$ & $32.00^{\mathrm{C}}$ & $29.00^{d}$ \\
\hline Root length $(\mathrm{cm})$ & $14.60^{c}$ & $14.80^{c}$ & $16.00^{\mathrm{b}}$ & $18.00^{a}$ & $14.90^{c}$ & $15.10^{c}$ & $16.80^{\mathrm{b}}$ & $18.20^{a}$ & $14.50^{c}$ & $14.70^{c}$ & $17.20^{\mathrm{b}}$ & $20.00^{a}$ \\
\hline Leaf length $(\mathrm{cm})$ & $8.58^{\mathrm{a}}$ & $8.53^{\mathrm{a}}$ & $8.50^{\mathrm{a}}$ & $8.50^{\mathrm{a}}$ & $8.40^{\mathrm{a}}$ & $8.40^{\mathrm{a}}$ & $8.40^{\mathrm{a}}$ & $8.40^{\mathrm{a}}$ & $8.50^{\mathrm{a}}$ & $8.50^{\mathrm{a}}$ & $8.00^{\mathrm{a}}$ & $8.00^{\mathrm{a}}$ \\
\hline Petiole length $(\mathrm{cm})$ & $7.38^{\mathrm{a}}$ & $7.40^{\mathrm{a}}$ & $7.35^{\mathrm{a}}$ & $7.40^{\mathrm{a}}$ & $7.40^{\mathrm{a}}$ & $7.40^{\mathrm{a}}$ & $7.40^{\mathrm{a}}$ & $7.40^{\mathrm{a}}$ & $7.40^{\mathrm{a}}$ & $7.35^{\mathrm{a}}$ & $7.40^{\mathrm{a}}$ & $7.35^{\mathrm{a}}$ \\
\hline Leaf area $\left(\mathrm{cm}^{2}\right)$ & $69.00^{\mathrm{a}}$ & $69.00^{a}$ & $68.75^{\mathrm{a}}$ & $68.75^{a}$ & $69.00^{a}$ & $68.75^{\mathrm{a}}$ & $68.50^{\mathrm{a}}$ & $68.50^{\mathrm{a}}$ & $69.25^{\mathrm{a}}$ & $69.00^{\mathrm{a}}$ & $69.00^{\mathrm{a}}$ & $68.75^{a}$ \\
\hline Vine length $(\mathrm{cm})$ & $275.00^{a}$ & $273.00^{a}$ & $252.00^{\mathrm{b}}$ & $243.00^{c}$ & $297.00^{a}$ & $298.25^{a}$ & $261.00^{\mathrm{b}}$ & $253.00^{c}$ & $293.50^{a}$ & $291.00^{b}$ & $260.00^{c}$ & $251.75^{\mathrm{c}}$ \\
\hline No of bulbils & $3.00^{\mathrm{a}}$ & $3.00^{\mathrm{a}}$ & $2.00^{\mathrm{b}}$ & $0.00^{c}$ & $5.00^{\mathrm{a}}$ & $5.00^{\mathrm{a}}$ & $3.00^{\mathrm{b}}$ & $1.00^{c}$ & $4.00^{\mathrm{a}}$ & $4.00^{\mathrm{a}}$ & $3.00^{\mathrm{b}}$ & $0.00^{\mathrm{C}}$ \\
\hline
\end{tabular}


Kalu et al.; ARRB, 36(1): 44-52, 2021; Article no.ARRB.61734

Table 3c. Morphological parameters of Dioscorea bulbifera treated with different concentrations of sodium chloride

\begin{tabular}{|c|c|c|c|c|c|c|c|c|c|c|c|c|}
\hline Parameter & $\begin{array}{l}\text { Line } 16 \\
0.00 \mathrm{mM}\end{array}$ & $100 \mathrm{mM}$ & $200 \mathrm{mM}$ & $300 \mathrm{mM}$ & $\begin{array}{l}\text { Line } 17 \\
0.00 \mathrm{mM}\end{array}$ & $100 \mathrm{mM}$ & $200 \mathrm{mM}$ & $300 \mathrm{mM}$ & $\begin{array}{l}\text { Line } 18 \\
0.00 \mathrm{mM}\end{array}$ & $100 \mathrm{mM}$ & $200 \mathrm{mM}$ & $300 \mathrm{mM}$ \\
\hline No of leaves & $40.00^{b}$ & $41.00^{\mathrm{a}}$ & $37.00^{c}$ & $33.00^{d}$ & $45.00^{b}$ & $46.00^{a}$ & $40.00^{\mathrm{C}}$ & $38.00^{\mathrm{d}}$ & $42.00^{a}$ & $42.00^{a}$ & $37.00^{b}$ & $35.00^{c}$ \\
\hline Root length (cm) & $14.80^{\mathrm{C}}$ & $15.20^{\mathrm{C}}$ & $16.60^{\mathrm{b}}$ & $18.00^{\mathrm{a}}$ & $15.00^{c}$ & $15.20^{\mathrm{C}}$ & $17.00^{\mathrm{b}}$ & $20.00^{a}$ & $15.20^{\mathrm{C}}$ & $15.20^{\mathrm{C}}$ & $18.00^{\mathrm{b}}$ & $21.00^{a}$ \\
\hline Leaf length $(\mathrm{cm})$ & $8.75^{\mathrm{a}}$ & $8.75^{\mathrm{a}}$ & $8.50^{\mathrm{a}}$ & $8.50^{\mathrm{a}}$ & $8.98^{\mathrm{a}}$ & $8.95^{\mathrm{a}}$ & $8.93^{\mathrm{a}}$ & $8.93^{\mathrm{a}}$ & $8.08^{a}$ & $8.05^{\mathrm{a}}$ & $8.05^{\mathrm{a}}$ & $8.03^{\mathrm{a}}$ \\
\hline Petiole length $(\mathrm{cm})$ & $7.35^{\mathrm{a}}$ & $7.40^{\mathrm{a}}$ & $7.40^{\mathrm{a}}$ & $7.38^{a}$ & $4.55^{\mathrm{a}}$ & $4.60^{\mathrm{a}}$ & $4.55^{\mathrm{a}}$ & $4.60^{\mathrm{a}}$ & $4.50^{\mathrm{a}}$ & $5.58^{a}$ & $4.50^{\mathrm{a}}$ & $4.55^{\mathrm{a}}$ \\
\hline Leaf area $\left(\mathrm{cm}^{2}\right)$ & $69.00^{\mathrm{a}}$ & $68.75^{\mathrm{a}}$ & $68.75^{a}$ & $68.75^{\mathrm{a}}$ & $69.75^{\mathrm{a}}$ & $69.75^{\mathrm{a}}$ & $69.75^{\mathrm{a}}$ & $69.75^{a}$ & $69.75^{\mathrm{a}}$ & $70.00^{a}$ & $69.75^{\mathrm{a}}$ & $69.75^{\mathrm{a}}$ \\
\hline Vine length $(\mathrm{cm})$ & $336.00^{a}$ & $338.00^{\mathrm{a}}$ & $312.00^{b}$ & $303.00^{c}$ & $354.00^{b}$ & $357.00^{a}$ & $338.00^{c}$ & $326.00^{d}$ & $344.00^{a}$ & $341.00^{b}$ & $318.00^{c}$ & $310.00^{d}$ \\
\hline No of bulbils & $4.00^{\mathrm{a}}$ & $4.00^{a}$ & $4.00^{\mathrm{a}}$ & $0.00^{\mathrm{b}}$ & $6.00^{a}$ & $5.00^{\mathrm{b}}$ & $3.00^{\mathrm{C}}$ & $1.00^{\mathrm{d}}$ & $8.00^{\mathrm{a}}$ & $7.00^{\mathrm{b}}$ & $5.00^{\mathrm{C}}$ & $2.00^{\mathrm{d}}$ \\
\hline Parameter & \multicolumn{2}{|c|}{$\begin{array}{l}\text { Line } 19 \\
0.00 \mathrm{mM}\end{array}$} & $100 \mathrm{mM}$ & \multicolumn{2}{|c|}{$200 \mathrm{mM}$} & $300 \mathrm{mM}$ & $\begin{array}{l}\text { Line } 20 \\
0.00 \mathrm{mM}\end{array}$ & $100 \mathrm{mM}$ & \multicolumn{2}{|c|}{$200 \mathrm{mM}$} & $300 \mathrm{mM}$ & LSD \\
\hline No of leaves & \multicolumn{2}{|c|}{$39.00^{a}$} & $38.00^{b}$ & \multicolumn{2}{|c|}{$33.00^{C}$} & $31.00^{\mathrm{d}}$ & $40.00^{\mathrm{a}}$ & $39.00^{b}$ & \multicolumn{2}{|c|}{$35.00^{c}$} & $35.00^{C}$ & 0.50 \\
\hline Root length (cm) & \multicolumn{2}{|c|}{$15.00^{d}$} & $16.00^{\mathrm{C}}$ & \multicolumn{2}{|c|}{$18.20^{\mathrm{b}}$} & $20.50^{a}$ & $14.90^{c}$ & $15.30^{c}$ & \multicolumn{2}{|c|}{$19.30^{\mathrm{b}}$} & $21.50^{\mathrm{a}}$ & 0.54 \\
\hline Leaf length $(\mathrm{cm})$ & \multicolumn{2}{|c|}{$8.75^{\mathrm{a}}$} & $8.50^{\mathrm{a}}$ & \multicolumn{2}{|c|}{$8.50^{\mathrm{a}}$} & $8.25^{a}$ & $8.25^{\mathrm{a}}$ & $8.25^{\mathrm{a}}$ & \multicolumn{2}{|c|}{$8.25^{\mathrm{a}}$} & $8.25^{\mathrm{a}}$ & NS \\
\hline Petiole length $(\mathrm{cm})$ & \multicolumn{2}{|c|}{$4.50^{\mathrm{a}}$} & $4.68^{\mathrm{a}}$ & \multicolumn{2}{|c|}{$4.50^{\mathrm{a}}$} & $4.55^{\mathrm{a}}$ & $4.55^{\mathrm{a}}$ & $4.55^{\mathrm{a}}$ & \multicolumn{2}{|c|}{$4.40^{\mathrm{a}}$} & $4.60^{\mathrm{a}}$ & NS \\
\hline Leaf area $\left(\mathrm{cm}^{2}\right)$ & \multicolumn{2}{|c|}{$70.25^{\mathrm{a}}$} & $70.25^{a}$ & \multicolumn{2}{|c|}{$69.75^{\mathrm{a}}$} & $69.75^{\mathrm{a}}$ & $69.75^{a}$ & $70.25^{\mathrm{a}}$ & \multicolumn{2}{|c|}{$69.75^{\mathrm{a}}$} & $69.75^{a}$ & NS \\
\hline Vine length (cm) & \multicolumn{2}{|c|}{$318.00 a$} & $315.00 b$ & \multicolumn{2}{|c|}{$306.00 \mathrm{c}$} & $294.00 d$ & $329.00 \mathrm{a}$ & \multirow{2}{*}{$\begin{array}{l}326.00 b \\
3.00 b\end{array}$} & \multicolumn{2}{|c|}{$310.00 c$} & $296.00 d$ & 2.10 \\
\hline No of bulbils & \multicolumn{2}{|c|}{$6.00 \mathrm{a}$} & $6.00 \mathrm{a}$ & \multicolumn{2}{|c|}{$3.00 \mathrm{~b}$} & $2.00 c$ & $4.00 \mathrm{a}$ & & \multicolumn{2}{|c|}{$2.00 \mathrm{c}$} & $0.00 \mathrm{~d}$ & 0.34 \\
\hline
\end{tabular}

Values are means of morphological parameters in Dioscorea bulbifera 
water by the root of the plant. To overcome this situation, the roots of the plant have to grow deeper into the soil to absorb water free from salt. Therefore, an increase in the root length of $D$. bulbifera could be regarded as a response to tolerate the sodium chloride stress condition. $[8,15]$ reported an increase in root length of Zea mays as a response to sodium chloride. An increase in root length of Prunus species treated with salt has also been reported [15].

The colour of the studied plant leaves at $200 \mathrm{mM}$ and $300 \mathrm{mM}$ concentration of $\mathrm{NaCl}$ was yellow. The leaf yellowing was more conspicuous at $300 \mathrm{mM}$ concentration of the sodium chloride. This is an indication of deficiency of some essential nutrients (sulphur, iron, magnesium and nitrogen) in the plants. Saline soils of higher concentration (above $200 \mathrm{mM}$ ) could lead to interference in the absorption of nutrients by the root hairs of the plants.

A reduction in concentrations of chlorophyll a and b in the treated plants especially at $300 \mathrm{mM}$ concentration of the salt was recorded. This implied that salinity caused a reduction in the chlorophyll $\mathrm{a}$ and $\mathrm{b}$ contents of the plant. Chlorophyll is an important material for photosynthesis. Reduction in chlorophyll content leads to a reduction in the rate of photosynthesis. This phenomenon accounts for the reduction in the growth of the treated plant.

\section{CONCLUSION}

Although salinity reduced the growth of $D$. bulbifera, in all the lines plants were able to tolerate the sodium chloride and its effect, regarded to grow until maturity and yielded bulbils except at $300 \mathrm{mM}$ concentration of the sodium chloride, where many of the plants did not yield at all. Given the foregoing, therefore, $D$. bulbifera could be listed as a moderately salttolerant plant.

Significant differences in plant growth were recorded among the lines. Lines $13-20$ showed more growth than the other lines. Lines $1-5$ which showed lower growth had spindle or elongated bulbils shape. Bulbils with a spindle or elongated shape could be said to be less tolerant of the salt treatment than the round or ovoidshaped.

\section{COMPETING INTERESTS}

Authors have declared that no competing interests exist.

\section{REFERENCES}

1. Kumar K, Kumar M, Kim SR, Ryu H, Cho YG. Insights into genomics of salt stress response in rice. Rice Journal. 2013;6:27.

2. Munns R, Tester M. Mechanisms of salinity tolerance. Annual Review of Plant Biology. 2008;59:651-681.

3. Gónmez-Bellot MJ, Álvarez $S$, Bañón $S$, Ortuño MF, Sánchez-Blanco MJ. Physiological mechanisms involved in the recovery of euonymus and laurustinussubjected to saline waters. Agricultural Water Management. 2013; 128:131-139.

4. Jampeetong $\mathrm{A}$, Brix $\mathrm{H}$. Effects of $\mathrm{NaCl}$ salinity on growth, morphology, photosynthesis and proline accumulation of Salvinianatans. Aquatic Botany. 2009; 91(3):181- 186.

5. Ramezani E, Sepanlou MG, Naghdi BH. The effect of salinity on the growth; 2011.

6. Shanker AK, Venkateswarly B. Abiotic stress in plants - Mechanisms and adaptations. In Tech Publisher, Rijeka, Croatia. 2011;428.

7. Wang WX, Vinocur B, Altman A. Plant responses to drought, salinity and extreme temperatures: towards genetic engineering for stress tolerance. Planta. 2013;218:114.

8. Esfen G. Morphology and physiology of Echiumomoenum Fisch. \& Mey. African Journal of Biotechnology. 2000;10(44): 8765-8773.

9. Rengasmy P. Soil processes affecting crop production in salt affected soils. Functional Plant Biology. 2010;37:613620.

10. Gharsallah C, Fakhfakh H, Grubb D, Gorsane F. Effect of salt stress on ion concentration, proline content, antioxidant enzyme activities and gene expression in tomato cultivars. AoB Plants. 2016;6.

11. Shahbaz M, Ashraf M. Improving salinity tolerance in cereals. Critical Review of Plant Science. 2013;32:237- 249.

12. Weisany W, Sohrabi $Y$, Heidari G, Siosemardeh A, Ghassemi-Golezani K. (). Changes in antioxidant enzymes activity and plant performances by salinity stress and zinc application in soybean (G/ycine max L.). Plant Omics Journal. 2012;5(2), 60-67. 
13. Zheng J, Zhao L, Shen B, Jiang L, Zhu A. Effects of salinity on activity and expression of enzymes involved in ionic, osmotic and antioxidant responses in Euryaemarginata. Acta Physiologiae Plantarum. 2016;38:70.

14. Jouyban $Z$. The effect of salt stress on plant growth. Technical Journal of
Engineering and Applied Sciences. 2012; 2(1):7-10.

15. Panuccio MR, Jacobsen SE, Akhtar SS, Muscolo A. Effect of saline water on seed germination and early seedling growth of the halophyte quinoa. AoB Plants. 2014; $6(2): 75-84$.

(c) 2021 Kalu et al.; This is an Open Access article distributed under the terms of the Creative Commons Attribution License (http://creativecommons.org/licenses/by/4.0), which permits unrestricted use, distribution, and reproduction in any medium, provided the original work is properly cited.

Peer-review history:

The peer review history for this paper can be accessed here: http://www.sdiarticle4.com/review-history/61734 